\title{
Criminologie
}

\section{Le crime organisé et la guerre aux stupéfiants : crise et réforme}

\section{Chet M. Mitchell}

Volume 22, numéro 1, 1989

Drogues et criminalités

URI : https://id.erudit.org/iderudit/017273ar

DOI : https://doi.org/10.7202/017273ar

Aller au sommaire du numéro

\section{Éditeur(s)}

Les Presses de l'Université de Montréal

ISSN

0316-0041 (imprimé)

1492-1367 (numérique)

Découvrir la revue

Citer cet article

Mitchell, C. M. (1989). Le crime organisé et la guerre aux stupéfiants : crise et réforme. Criminologie, 22(1), 41-65. https://doi.org/10.7202/017273ar
Résumé de l'article

War is a form of competition and the drug wars are no exception to this definition. Drug wars are actually classic illustrations of competitors abusing the legal process to define their own drug trading as lawful while characterizing their competitor's behaviour as "crime". Successive American federal administrations extended the drug wars through a combination of military assistance, financial pressure and secret agreements. These aggressions are the real abuses aimed at third world cultures. Since Americans purchase $60 \%$ of all illicit drugs and finance more than $90 \%$ of the police action against the trade, drug legalization drug crusade. On the other hand, even if drug legalization makes sense the U.S. federal government will not necessarily act sensibly. An alternative possibility is reform outside the U.S. capable of generating a competitive crises internationaly.
Ce document est protégé par la loi sur le droit d'auteur. L'utilisation des services d'Érudit (y compris la reproduction) est assujettie à sa politique d'utilisation que vous pouvez consulter en ligne.

https://apropos.erudit.org/fr/usagers/politique-dutilisation/ 


\begin{abstract}
War is a form of competition and the drug wars are no exception to this definition. Drug wars are actually classic illustrations of competitors abusing the legal process to define their own drug trading as lawful while characterizing their competitor's behaviour as "crime». Successive American federal administrations extended the drug wars through a combination of military assistance, financial pressure and secret agreements. These aggressions are the real abuses aimed at third world cultures. Since Americans purchase $60 \%$ of all illicit drugs and finance more than $90 \%$ of the police action against the trade, drug legalization drug crusade. On the other hand, even if drug legalization makes sense the U.S. federal government will not necessarily act sensibly. An alternative possibility is reform outside the U.S. capable of generating a competitive crises internationaly.
\end{abstract}

\title{
1. CROISADES CONTRE LES STUPÉFIANTS
}

\section{A. LES DROGUES, UNE MENACE INTERNATIONALE}

La guerre est une forme de compétition et les guerres contre la drogue ne constituent pas l'exception à la règle car elles résultent de rivalités professionnelles, commerciales et culturelles. Tout au long de l'histoire, des groupes se sont disputé le contrôle des drogues et, puisque la victoire dépend surtout de la puissance politique, les drogues commencent habituellement leurs carrières sociales sous forme de médicaments, d'objets de culte ou de prérogatives royales (Szasz, 1974). Afin de protéger leur hégémonie, les magnats du trafic de stupéfiants doivent combattre les petits concurrents qui vendent moins cher, empiétent sur leur marché ou menacent les privilèges que la drogue leur confère. La lutte aux drogues commerciales est aussi une constante historique, et ce, en raison du rôle central que le thé, le rhum, le tabac, le café, l'opium, la coca et autres drogues ont joué dans la fondation de plusieurs empires commerciaux/coloniaux (Rublowsky, 1974; Goode, 1984). Les drogues demeurent parmi les plus importantes matières premières échangées sur le marché international, là où le café occupe, à lui seul, le deuxième rang (en valeur brute) après le pétrole (Bates \& Lien, 1985). Si le commerce des drogues licites, telles le tabac, l'alcool

* Texte traduit de l'anglais par Nathalie Cadieux. K1S 5B6.

** L'auteur est professeur de droit à l'Université Carleton, Ottawa, Ontario, 
et les produits pharmaceutiques, est dominé par l'Europe et l'Amérique du Nord, les drogues illicites, elles, proviennent de pays moins industrialisés. Par le biais de cartels, de tarifs douaniers, de boycottages et de guerres, les trafiquants de drogue cherchent à se donner un avantage relatif sur leurs rivaux. Enfin, la principale compétition se situe au niveau culturel. Les cultures transcendent les entreprises et même les nations et propagent invariablement leurs propres mythes, symboles, langues et modes. Les peuples ont tendance à se méfier des articles qui sont étrangers à leur culture: aliments, modes, drogues ou autres sont souvent rejetés comme étant malsains (Satinder, 1980, p. 125). Après 1906, les législateurs canadiens s'attaquaient aux consommateurs d'opium, de cocaïne et de marijuana, principalement parce que ces drogues étaient «étrangères» (Boyd, 1984; Solomon et Green, 1982). Tout comme les aliments tabous, les drogues prohibées ne sont essentiellement pas plus néfastes que l'alcool ou le tabac (Mitchell, 1986a). Dans des contextes historiques différents, les guerres contre les stupéfiants pourraient maintenant être dirigées contre la nicotine et le tabac et être tout aussi justifiées.

Le statut juridique de toutes les drogues hors-la-loi découle de ces trois modèles évolutifs (Cloyd, 1982; Walker, 1981). Avant que la conquête espagnole ne détruise leur pouvoir, la noblesse et les prêtres incas monopolisaient la coca (McNicoll, 1983). Après son arrivée relativement récente en Amérique du Nord, la cocaïne fut faussement liée à la violence noire et devint l'objet d'une lutte de pouvoir entre pharmaciens, médecins et fabricants de médicaments brevetés (Murray, 1988; Grinspoon et Bakalar, 1976). La marijuana, elle, apparut historiquement sous l'autorité des prêtres indiens. Néanmoins, au moment de son interdiction en Amérique du Nord quelques milliers d'années plus tard, le cannabis fut associé aux immigrants pauvres du Mexique, aux Noirs des États du Sud et à la main-d'oeuvre agricole migrante. Introduit en Chine vers l'an 800 avant J.-C. par des commerçants arabes, l'opium y était mangé pour ses vertus médicinales et aussi comme divertissement aristocratique (MacFarlane, 1986; Fort, 1969). Après de nombreuses mésaventures avec la loi chinoise, l'opium devint, à partir de 1890, la cible des législateurs nord-américains, qui entreprirent une campagne raciste contre les immigrants et les cultivateurs chinois.

La carrière de l'opium en Chine est exemplaire et occupe le centre des préoccupations actuelles. Lorsque les marchands portugais apportèrent du tabac en Chine, l'Empire du Milieu était alors le plus grand et le plus populeux de la planète. Les gouvernements chinois étaient formés de professionnels et d'intellectuels plutôt hostiles aux importations 
étrangères. Ainsi, lorsque l'usage récréatif du tabac se répandit rapidement, l'empereur n'hésita pas à l'interdire en 1644. Par la suite, les fumeurs se mirent à camoufler leur usage de drogue illicite en fumant leur tabac mélangé à de l'opium. La prohibition du tabac a donc déclenché l'habitude de fumer l'opium et a contribué à populariser son emploi, ce qui mit en danger son contrôle médical. Enfin, en 1729, l'usage non médical de l'opium fut interdit.' (Le monopole médical sur certains psychotropes, lequel fit son apparition au Canada avec la cocaïne en 1908 , profite aux médecins mais non à la santé publique.) (Mitchell, 1986b). Les lois chinoises sur les opiacés engendrèrent des prix de marché noir qui attirèrent les contrebandiers britanniques. À partir du moment où les dirigeants mercantilistes chinois décidèrent que l'importation illégale d'opium nuisait à la balance commerciale, on fit respecter les lois anti-importation. Les Britanniques ripostèrent par les armes durant les guerres de l'opium de $1839-42$ et $1856-58$, et pendant un demi siècle, les Chinois connurent une série de défaites humiliantes aux mains des Européens (Garraty et Gay, 1972). Si bien que les nationalistes chinois vinrent à considérer l'opium comme le bouc émissaire et symbole de la domination européenne, une excuse parfaite pour justifier l'échec du gouvernement (Musto, 1973).

À ses débuts, vers 1890 , l'opposition américaine au commerce de l'opium fut officiellement attribuée à un souci humanitaire envers les Chinois (Taylor, 1969). Pourtant, cette noble cause oubliait les millions de Chinois fumeurs de tabac et détonnait avec le préjugé anti-oriental des Américains pour qui les immigrants chinois fumeurs d'opium étaient des «porteurs de maladies» transmettant encore une autre «peste venant d'Asie» (Epstein, 1977, p. 27). ${ }^{2}$ En fait, la politique américaine sur les opiacés était plutôt dictée par l'égoïsme compétitif des Américains dans le domaine de l'expansion coloniale (la prise des Philippines), ainsi que par leur volonté d'obtenir un avantage sur les Britanniques et des concessions commerciales de la part des Chinois

1. McNicoll suggère que le gouvemement chinois a prohibé l'usage non-médical de l'opium parce qu'il a «rapidement compris» les dangers de «l'assuétude» (p. 5), Cette affirmation est un cas criant de révisionnisme historique puisque en 1729 l'opium faisait partie des coutumes chinoises depuis plus de mille ans, c'est-à-dire depuis la Dynastie T'ang (618-907 av. J.-C.), Cf. Hess (1965, p. 13)

2. Le thème du péril jaune faisait déjà partie de l'atmosphère sociale de l'époque. À partir de 1913, les populaires romans du Docteur Fu Manchu de Sax Rohmer imaginaient un complot oriental visant à réduire l'Occident à l'esclavage au moyen de drogues. Dans les années 30, les opiacés étaient fermement associés à l'esclavage, à l'invasion étrangère, à l'immoralité, au crime, à la paresse et à la maladie. En 1928, une émission du réseau NBC comparait les opiomanes à des lépreux et les rendait responsables d'une «alarmante vague de crime». (Baridon, 1970, p. 2) 
(Stephens, 1987; Morgan, 1981; Walker, 1981). En 1905, les entreprises américaines se trouvèrent confrontées à un embargo commercial organisé par les marchands chinois. Ces derniers protestaient contre les mauvais traitements infligés aux Chinois établis aux États-Unis. C'est donc pour apaiser leur colère que les États-Unis proposèrent la Conférence de Shanghaï sur l'opium (Musto, 1973).

Après 1900, les législateurs nord-américains ne luttaient pas contre les drogues en général, mais bien contre les drogues produites à l'étranger et ne faisant pas partie de la culture courante. L'enjeu consistait à bâtir un monde sécuritaire pour une société qui fonctionnerait selon le modèle tabac-alcool-médicaments et à optimiser l'exportation de «bonnes» drogues en dépit des maladies et des morts prématurées qu'elles causent (Silverman, Lee et Lydecker, 1982; White, 1988). Les cargaisons de tranquillisants à destination du Tiers-Monde se font donc plus volumineuses sans qu'on n'y voie de problème, alors que les ventes de drogues dans l'autre direction (à l'exception du thé et du café) sont dénoncées en tant qu'actes de terrorisme. Il s'agit là d'une stratégie courante pour s'arroger un privilège : on criminalise l'entreprise concurrente.

Les lois anti-opiacés établirent le modèle législatif du Canada et des États-Unis : confier le contrôle des drogues à la police, harceler les médecins; criminaliser les consommateurs; appliquer la loi sélectivement auprès des minorités; blâmer les fournisseurs étrangers pour la corruption, la criminalité et le climat insalubre engendré par la loi; coopter des armées internationales et profiter des crises pour intensifier la guerre aux drogues (Zinberg et Robertson, 1972). La chasse aux sorcières de la "guerre froide» inaugurait une période particulièrement violente et des sanctions plus sévères allant jusqu'à la peine de mort. Elle répandait le mythe du junkie criminel et associait les drogues illicites à la domination du monde par le communisme (Ray, 1978, pp. 3558). Pendaant des décennies, les dirigeants nords-américains se sont dépeints assiégés par la menace de drogues «contaminantes» venant de continents étrangers. Divers ennemis des É.-U., y compris, en 1916, Kaiser Wilhelm et sa cocaine, offraient des cibles toutes choisies pour la propagande américaine, ainsi que le décrit Epstein (1977, p. 81):

Pendant la deuxième guerre mondiale, «Les militaristes japonais» ont été accusés de trafic de drogue; les nationalistes iraniens ont été montés en épingle en 1951...; après la prise de pouvoir de Castro en 1957, Cuba a été cité comme fournisseur de marijuana aux É.-U.; la Chine communiste a été accusée de mettre au point «un plan, échelonné sur vingt ans, visant à répandre l'assuétude 
parmi les peuples libres»; l'Union soviétique et ses pays-satellites ont été qualifiés de... principaux trafiquants d'héroïne; enfin, le Vietnam du Nord a été ajouté en 1962 à la liste des contrevenants...

La croisade contre la drogue a si bien déguisé la nature de son combat contre les cultures étrangères, les idéologies concurrentes ou les immigrants, que ses soldats connaissent peu les drogues illicites ou alors ne s'en soucient guère. ${ }^{3}$ Smart et Murray (1984, p. 34) suggèrent charitablement que les projets internationaux de lutte aux drogues ne s'appuient pas toujours sur des critères scientifiques. Mais en réalité, de tels projets sont irrémédiablement biaisés puisqu'ils définissent la «toxicomanie» comme étant l'usage de n'importe quelle drogue provenant d'une autre culture (Pan et Bruun, 1979). Selon les définitions des Nations Unies, la Jamaïcaine pauvre qui boit du thé au ganja deux fois par semaine est une toxicomane criminelle, tandis que le diplomate alcoolique et le politicien qui fume comme une cheminée ne le sont pas. La majeure partie de l'information traitée par les législateurs provient d'agences chargées d'appliquer les lois et déterminées à étendre leur croisade jusqu'au sein des autres forces policières. ${ }^{4}$ Par exemple, la lutte internationale anti-cannabis était dirigée par la police sud-africaine et endossée par les autorités égyptiennes qui, en 1925 à Genève, avaient déclaré le haschich responsable de presque toute la délinquance en Égypte (Bonnie et Whitebread, 1970). Sans doute le plus influent responsable de l'application d'une loi a été $\mathrm{H}$. Anslinger, directeur du U.S. Narcotics Bureau pendant 32 ans. Pourtant, un chercheur contemporain admet maintenant que les opinions d'Anslinger sur les stupéfiants ressemblaient «aux divagations d'un fou» (Inciardi, 1986, p. 22).

La documentation internationale sur les stupéfiants se résume en gros à des rapports de police et à des descriptions sans commentaires critiques (Robertson, 1977; Bresler, 1980). L'ouvrage de Chatterjee Legal Aspects of International drug Control présume sans apporter

3. Voir, par exemple, Drug Enforcement, un journal publié par le Département américain de la Justice. La plupart des articles démontrent une acceptation totale du mythe de la «drogue dangereuse», déclarant, sans preuve à l'appui, que ces drogues «sont incontestablement de graves dangers pour la population» (1982, Vol, $9 \# 2$, p. 2), Quand ces articles prouvent leur «dangerosité» par des expériences médicales, celles-ci sont biaisées et non-scientifiques. Cohen (ib., p. 10) dénonce «l'auto-indulgence narcissique» et prétend que la cocainne est «la drogue rêvée de l'hédoniste». Dans un texte de 1978 (Vol. 5, \#l, p. 17), on soupçonne le PCP «de déchaîner les pires démons de l'âme humaine».

4. Parce qu'il y a déjà eu un lien étroit entre l'opiomanie et la criminalité, les bureaux de stupéfiants se sont multipliés et sont devenus un complexe réseau d'information sur l'activité clandestine. Ces bureaux ont aussi permis aux Américains d'exporter du personnel et de l'expertise. 
aucune preuve que «l'on peut difficilement trop insister sur la nécessité de supprimer le trafic illicite de drogues» (p. vii). En général, on attribue les effets de la prohibition à la nature des drogues (Taylor, 1969). En confondant les effets de la drogue avec les répercussions des lois, on permet aux initiateurs de la croisade de ne plus prendre la responsabilité des conséquences de leurs actions. On peut alors changer le noir en blanc comme lorsque Waddell (1970) compare la croisade anti-drogue à la lutte pour abolir l'esclavage. Pourtant, les anti-esclavagistes luttaient pour l'égalité des races tandis que les forces anti-stupéfiants encouragent l'inégalité juridique en renforcant le mythe selon lequel «drogués» et «revendeurs de drogue» sont des déchets de la société indignes des protections juridiques normales (Freemantle, 1985; Lidz et Walker, 1980, p. 78). Toutefois, depuis 1970 , on peut lire un certain nombre d'analyses critiques sur les guerres contre la drogue (McCoy, 1972; Lamour et Lamberti, 1972; Gooberman, 1974; Wisotsky, 1986; Trebach, 1987).

\section{B. LES CRIMES CONTRE L'HUMANITÉ}

Comme l'art, les croisades se terminent probablement toutes dans l'excès. Pour certains, les drogues illicites sont les «têtes de l'Hydre du mal, les corrupteurs des valeurs traditionnelles, les destructeurs de la moralité, l'antithèse du style de vie américain» (Johnson, 1986). Des opinions similaires ont entaché les résolutions de l'ONU en 1984 lorsque l'Assemblée qualifia le trafic des stupéfiants de «grave crime international contre l'humanité» et proposa un nouvel accord selon lequel tous les signataires s'engageaient à imposer «des peines criminelles rigoureuses» aux contrevenants (UN Chronicle, 1984). Le préambule critique les «visées commerciales méprisables» des trafiquants et signale que les drogues sapent les forces militaires. Le secrétaire-général Pérez de Cuéllar déclare que le trafic illicite de stupéfiants est «un des maux les plus malsains et les plus contagieux de notre époque» (ib.).

L'exemple de l'Amérique du Nord a fait en sorte que les lois sur les stupéfiants sont désormais perçues comme des symboles de modernité. Dans la foulée de la technologie occidentale, de la musique pop, des modes et des méga-projets souvent mal planifiés, certains gouvernements du Tiers-Monde ont suivi le mouvement anti opium. ${ }^{5}$ Les gou-

5. Dès 1928 , des représentants égyptiens se faisaient un honneur de fonder un Bureau central des stupéfiants. Ces institutions ont l'heur de plaire aux gouvemements de pays en voie de développement. En effet, ceux-ci ont un penchant pour les palais, les arénas gigantesques et autres méga-projets souvent mal planifiés, tel le barrage d'Aswan. 
vernements de l'Iran, du Japon, de l'Afrique du Sud et maintenant de la Malaysie démontrent leur zèle humanitaire en incarcérant ou en exécutant les trafiquants de stupéfiants. Avec son Dangerous Drug Act (loi sur les drogues dangereuses), le gouvernement de la Malaysie a pendu des douzaines de gens et des centaines d'autres attendent encore leur jugement ou leur exécution. ${ }^{6}$ Ainsi, à côté des sévères politiciens malais, les législateurs fédéraux du Canada font figure de piètres soldats dans cette guerre contre «les maux les plus malsains et les plus contagieux de notre époque». En effet, le Parlement n'a imposé qu'une peine de sept ans de prison pour importation de drogue et, pire encore, la Cour Suprême du Canada a jugé cette peine inconstitutionnelle. ${ }^{7}$

L'opinion classique sur le sujet ressemble autant à du racisme, à de l'ignorance et à de la propagande de guerre que pendant les années 30 . Selon Mills (1986) les É.-U. sont la principale victime des «attaques à la drogue» du Mexique, de la Colombie, de la Bolivie et du Pérou, lesquels sont tous des pays qui méritent davantage de représailles militaires que la Libye. La majorité des revues d'actualité approuve la ligne dure contre les stupéfiants, surtout le U.S. News and World Report. Sous le titre «Narcotics: Terrors' New Ally» (La drogue, nouvelle alliée du terrorisme) le texte se lit comme suit :

De toutes les alliances, elle est la plus sacrilège. C'est un mariage malveillant entre deux fléaux, parmi les plus destructeurs et les plus craints qui frappent la société moderne: le terrorisme et la drogue... Les narco-profits offrent une manne de possibilités à ceux qui cherchent à déstabiliser les démocraties, à répandre l'anarchie et à exporter la révolution.

(4 mai 1987, pp. 30-37)

Deux autres publications de cette même revue méritent d'être citées. Dans l'une (8 sept. 1986, p. 69), un procureur (district attorney) de New-York prétend que le «flot de drogues qui afflige cette nation est un acte de terrorisme contre notre peuple... nous devons... éliminer ce cancer». Le deuxième article allègue que la drogue n'est plus un «danger limité aux rues» mais une menace à la sécurité nationale (28 juillet 1986, pp. 48-55):

6. Après que deux Australiens furent pendus en 1986, incitant le premier ministre de l'Australie à se plaindre de la «barbarie malaysienne», un ministre du gouvernement malaysien déclara: «nous n'avons pas pendu d'Australien... nous avons pendu des trafiquants de drogue» (Far Eastern Eco. Rev., juillet 1986). De toute Évidence, les trafiquants sont des pirates sans patrie et aucun pays décent se méfiant de la drogue, ne devrait les réclamer.

7. Smith, cf. The Queen (1987), 34 CCC (3d) 97 (SCC) Sous-section 5(2) du Narcotic Control Act (Loi sur les stupéfiants) RSC 1970, C.N-1, prévoit encore la peine maximale d'emprisonnement à vie. 
Les raids militaires U.S. contre les caîds de la drogue mettent en relief l'état d'alarme qui règne dans les hautes sphères de la Maison Blanche. On s'inquiète de l'effet des drogues sur le peuple américain... personne ne semble en sécurité, ni à l'abri des complots malhonnêtes.

Les journalistes canadiens reprennent en choeur le discours américain, surtout la revue Maclean's qui exploite la guerre aux stupéfiants et nous avertit qu'en «Amérique du Nord, où l'héroünomanie se répand à un rythme alarmant et fait grimper les statistiques sur les crimes violents et crapuleux, on conçoit aisément que la meilleure solution consiste à détruire le pavot somnifère à sa source» (Burstein, 1982).

Les déclarations officielles laissent le même goût amer d'hypocrisie enflammée. Ainsi, le secrétaire d'État Schultz a comparé les trafiquants à des «pirates» bien que ce soient les navires américains qui ouvrent le feu sur les cargos de marijuana non armés dans les eaux internationales. Richard Nixon, durant sa présidence en 1972, donna le ton à ce type de discours quand il expliqua à un auditoire international (Ray, 1978, p. 48):

Les hommes et les femmes qui font le commerce international de l'hérö̈ne menacent non seulement les Américains, mais l'humanité tout entière. Ces gens sont littéralement les marchands d'esclaves de notre époque. Ils trafiquent la mort vivante. Il faut les chasser jusqu'aux confins de la terre, jusqu'à ce qu'il ne leur reste plus, dans quelque nation que ce soit, un seul lieu où mener leur opération.

Cette rhétorique reflétait les plans américains d'intensification de la croisade.

\section{INTENSIFICATION DE LA CROISADE}

Les administrations fédérales américaines intensifièrent les guerres anti-drogue grâce à une combinaison d'aide militaire, de pressions financières et d'accords secrets. Au cours du 92e Congrès, on présenta plus de cent projets de loi; beaucoup visaient à couper l'aide accordée aux «mauvais» gouvernements. (McNicoll, 1983, p. 69). Déjà, le gouvernement américain modifie le statut (décertifie) des pays qui ne luttent pas contre les stupéfiants avec assez d'enthousiasme. Lorsqu'un pays est décertifié, comme l'ont été l'Iran, la Syrie et l'Afghanistan, il perd l'aide américaine et ses privilèges commerciaux. (TIME, 7 mars 1988). Nixon fournit des policiers et de l'équipement aux pays participant à la croisade, puis Reagan intensifie davantage le conflit en dépensant 1,5 millards de dollars par année et en irnpliquant 
le département de la Défense (Wisotsky, 1986). D'anciens militaires sont recrutés pour servir dans la guerre aux stupéfiants. Par exemple le major E. Merwin, ex-membre de l'Armée de l'air (U.S. Air Force), engagé par le State Department's Bureau of International Narcotics Matters (BINM) pour diriger les Léopards, une brigade bolivienne antistupéfiants.

Les sept agences fédérales américaines qui combattent à l'étranger concentrent leurs efforts à détruire les plantations et à leur substituer d'autres cultures (Lee, 1985). Bien que les subventions aident à limiter la production de tabac de Virginie, elles sont inefficaces sur le marché noir de l'Amérique du Sud. Au Pérou et en Colombie, le coca* rapporte dix à quinze fois plus d'argent que n'importe quelle autre culture. La destruction des cultures par la force devient donc la solution logique, d'où le fait qu'en 1983 les gouvernements de la Bolivie et des É.-U. signaient des accords secrets selon lesquels les É.-U. payèrent 7,5 millions de dollars à la Bolivie pour qu'elle limite la culture du coca. Sur 200000 hectares de coca, 200 furent officiellement détruits (TIME, 4 août 1986). La disponibilité de la drogue sur le marché américain permet de mesurer le succès de la démarche américaine: de 1976 à 1984 les cargaisons de cocaïne sont passées de 19 à 137 tonnes métriques; en termes réels, le prix de la marijuana est plus bas aujourd'hui qu'il y a vingt ans, depuis que les saisies ont donné naissance à une solide industrie nationale; enfin, la disponibilité de l'héroïne reste stable, et ce, malgré 70 ans d'action policière (Wisotsky, 1986; Gooberman, 1974; Science 14 nov. 1975, p. 638). On peut difficilement bloquer la «source» parce que la source déménage. La culture du coca s'est déplacée en Équateur, au Panama et au Brésil et pourrait encore s'exiler jusqu'en Indonésie, à Madagascar, en Guyane, au Sri Lanka ou à Java (Lee, 1985). Et même si les É.-U. défoliaient presque tout le Tiers-Monde, il resterait encore des laboratoires clandestins capables de produire des substituts synthétiques à bon marché.

Malgré le bilan négatif et les sombres expectatives, les responsables américains recommandent davantage d'interventions policières; tout comme ils le faisaient au Vietnam alors que les études sérieuses ne correspondaient pas à l'optimisme affiché par le gouvernement. On peut dresser d'autres parallèles avec le Vietnam: encore une fois, les chefs politiques ne parviennent pas à assigner des objectifs réalisables ou à établir des critères de réussite clairs et, en conséquence, ils sont

* N. du T.: Coca est masculin quand il désigne l'arbrisseau, et féminin lorsqu'il s'agit de la substance extraite de la feuille. 
incapables de discipliner les fonctionnaires qui dirigent ces programmes voués à l'échec. À mesure qu'on alloue de l'équipement et du personnel à la guerre anti-drogue, on requiert de la protection supplémentaire. Comme avec la menace communiste, la route de la guerre est pavée de rhétorique déshumanisante. Enfin, on fait peu de cas de la résistance populaire aux agressions américaines. Après son frustrant tour de service avec les Léopards boliviens, Merwin était convaincu qu'avec les bonnes méthodes il pourrait juguler la production de cocaïne en quelques semaines. Merwin croit que :

La seule chose qui fonctionnera, c'est la force. Je vois cela comme une guerre. C'est une menace à notre sécurité nationale, au même degré qu'une menace militaire... [Nous devons] internationaliser une force de frappe arrêter les principaux trafiquants... Je détruirais leurs outils de production... Je mettrais le feu à leurs maisons...(Kline, 1987)

Merwin, cependant, ne tient pas compte du peuple ni de sa réaction aux forces de frappe. Selon les sondages, la plupart des citoyens de la Colombie, de la Jamaïque, du Pérou et de la Bolivie s'opposent à la destruction des cultures de coca (Lee, 1985). Les officiers démissionnent de l'armée plutôt que de déraciner les arbrisseaux; il est d'ailleurs possible que certains défendraient activement les producteurs de drogues bien armés contre les forces militaires américaines. Les guérillas, dont certaines seraient subventionnées par l'Union Soviétique, rendraient vains les efforts des É.-U., et allongeraient les «quelques semaines» de Merwin en années, en milliers de morts et de blessés et, finalement, en défaite, conformément aux modèles du Vietnam et de l'Afghanistan. L'impotence de l'action policière amena un agent de la DEA (Drug Enforcement Administration) à déclarer que si «vous voulez vraiment empêcher les drogues de sortir du Liban, vous devriez envoyer des B-52, pas des policiers» (Favret, 1986). Mais par ce raisonnement, il oublie que pour cibler les stupéfiants, l'action militaire n'est pas davantage efficace.

\section{OPPOSITION, RÉFORME ET RÉVOLUTION}

\section{A) AIDE AUX PAYS ENNEMIS}

Avec le temps, les lois imprécises se déstabilisent. En effet, leurs résultats contre-productifs créent des pressions appelant l'abrogation ou la réforme. La croisade anti-drogue crée des pressions de ce genre en aidant les ennemis et en décourageant les amis.

Les ennemis s'enrichissent parce que la croisade offre des possibilités de revenus aux gouvernements hostiles et aux révolutionnaires. 
C'est grâce aux lois nord-américaines sur les drogues que les dirigeants de Cuba, de la Bulgarie et du Nicaragua peuvent profiter des conditions du marché noir. Wisotsky (1986, p. 162) explique que «jamais les trafiquants de stupéfiants, les narco-terroristes, ni les gouvernements hostiles ne sont arrivés à concevoir un système mieux adapté à la promotion de leurs intérêts». Ultérieurement, les prohibitionnistes extrémistes se retrouvent coincés par les modérés qui s'aperçoivent que les nations ennemies et le crime organisé sont les premiers à appuyer la prohibition. Les bureaux des stupéfiants fonctionnent comme des comités de contrôle de la vente minés par la corruption: ils subventionnent les groupes divergents qui, à cause de leur éloignement, de leur statut de victime de persécution ou de leur anti-américanisme paient moins cher leur entrée dans le monde clandestin du trafic de drogue (Blackwell, 1988). Mais les narco-profits sont un couteau à deux tranchants, puisqu'en finançant l'insurrection armée, ils réduisent l'ardeur des révolutionnaires. Khun Sa, chef de l'Armée unie du Shan, est un «caïd de l'opium» qui cherche à former un État Shan indépendant. Il donne maintenant son appui au Parti communiste Birman, un allié circonstanciel. Autrefois un groupuscule militant, l'armée de l'indépendance de Khun Sa est aujourd'hui une entreprise florissante qui dépend des lois étrangères sur la drogue pour maintenir un niveau de vie élevé (TIME, 8 mars 1982 et 17 janvier 1983). De même, l'opulence apportée par les stupéfiants peut transformer les révolutionnaires latino-américains comme le M-19 colombien et le Sentier Lumineux péruvien en représentants de la bourgeoisie.

Aujourd'hui, les fonctionnaires américains ne déclarent plus que les principaux acteurs comme l'Union Soviétique conspirent à dominer le monde par le biais des stupéfiants. Toutefois, un ancien responsable du BINM croit que les Soviétiques «appuient les criminels internationaux et les organisations clandestines. C'est à leur avantage : ils savent que nous devons consacrer d'énormes ressources pour régler les problèmes de drogue et faire appliquer les lois» (U.S. News \& World Report, 4 mai 1987). Quoi qu'il en soit, la guerre que les Soviétiques mènent chez eux contre l'opium, le cannabis et la cocainne ne peut que réduire leurs chances de tirer profit des croisades de l'Occident. Selon le ministre des Affaires intérieures, A. Vlasov, «le combat contre la drogue et contre les crimes qui y sont rattachés est devenu l'une des tâches principales de notre ministère" (TIME, 19 jan. 1987). Les Soviétiques et les Américains sont en quelque sorte des alliés naturels dans cette guerre puisque, en ce qui a trait aux stupéfiants, tous deux partagent la même culture et le même impérialisme envers le TiersMonde. Si les conflits idéologiques sont délaissés au profit de l'écono- 
mie, on peut prévoir une coopération accrue des super-puissances dans la lutte aux stupéfiants (Globe \& Mail, ler sept. 1987).

\section{B. RANCUNE DES PAYS ALLIÉS}

Par le passé, la politique étrangère américaine a transformé certains pays alliés et partenaires commerciaux (Cuba, Vietnam, Chine) en ennemis (Pastor, 1986). La politique actuelle sur les drogues éveille elle aussi l'hostilité des alliés car elle corrompt les fonctionnaires, aggrave la violence civile et dresse une barrière entre l'élite dirigeante et la majorité paysanne.

La déstabilisation serait minimale si les É.-U. suivaient l'exemple isolationniste de la Chine des années 1800 et se contentaient de faire leur guerre contre l'opium à la maison, ou encore si, comme l'Arabie Saoudite, ils s'abstenaient de partir en croisade internationale contre les substances prohibées (Bradley, 1986). Dans ces conditions, les gouvernements des pays exportateurs de drogue suivraient l'exemple de l'Angleterre en Inde et légaliseraient la production de stupéfiants, à tout le moins pour fins d'exportation. Car une fois l'exportation rendue légale, les producteurs n'auraient plus besoin d'entretenir une armée privée ou de graisser les policiers pour avoir de la protection. Si elle était légale, l'industrie de la cocaïne en Amérique latine ne serait pas plus violente que celle du café. Mais parce que les É.-U. exportent leur guerre contre les drogues, les producteurs du Tiers-Monde opèrent en eaux troubles et leurs gouvernements sont tiraillés entre les obligations nationales et les pressions américaines. Morales élabore:

Au niveau international, le Pérou, pays producteur de cocaine, fait face à un sérieux dilemne. Le gouvernement veut protéger les coutumes de la majorité vivant dans les Andes et il blâme la société de consommation pour l'existence dé la cocaïne au Pérou, mais en même temps, il accepte que les É.-U. lui imposent de nouvelles politiques de production de coca.

En se pliant aux demandes américaines relatives à la destruction des cultures et à l'extradition des citoyens aux É.U., les gouvernements étrangers ne servent pas les intérêts de la majorité (Ottawa Citizen, août 1986). D'ailleurs, au cours des années 50 , les institutions démocratiques ont apparemment mieux survécu dans les pays qui n'étaient pas protégés de la contagion communiste par les É.-U. (Muller, 1985). Le personnel gouvernemental péruvien, typiquement européen d'origine et américain de formation, néglige les intérêts des paysans à cause de l'aide américaine, des prêts étrangers, des décisions du FMI et des investissements des sociétés commerciales dans le pétrole, les minéraux 
et l'industrie (Strug, 1985). Les régions occupées par les paysans, où la drogue est cultivée, reçoivent peu d'aide et sont aliénées des gouvernements centraux lointains à cause des assauts militaires, de l'épandage d'herbicide, de la corruption officielle et des conflit raciaux (Haggani, 1986). Des régions entières du Pakistan, de la Birmanie, du Pérou, de la Colombie et de la Bolivie fonctionnent ainsi sans contrôle gouvernemental (Inciardi, 1986; Lee, 1985).

L'application des lois sur les drogues dans les pays producteurs constitue un cas exagéré de «capture du régulateur», procédé par lequel les industries contrôlées établissent, avec leur régulateur, des liens étroits et réciproquement profitables. Une des conséquences importantes de cette capture du régulateur est le nombre accru de représentants gouvernementaux étrangers accusés par les applicateurs de la loi américaine. On enquête sur la police mexicaine, sur le colonel haïtien Jean-Claude Paul et sur le premier ministre des Bahamas; le ministre en chef de Turks et Caicos purge une sentence de huit ans dans une prison américaine et le général Manuel Noriega, dirigeant de facto du Panama, est accusé par un procureur américain (U.S. Attorney) de conspiration en vue d'importer de la cocaïne et de la marijuana aux É.-U. (Lee, 1985; TIME, 1988). Certains responsables américains étaient au courant des activités illicites de Noriega depuis 1972 (trafic de stupéfiants, chantage en échange de protection) mais continuaient à l'appuyer en échange du coup de main qu'il donnait aux 10000 militaires américains présents au Panama (TIME, 15 fév. 1988). En criminalisant les représentants étrangers, on risque de voir naître des ressentiments, entre autres parce que certains objecteront que s'ils avaient les ressources nécessaires, ils pourraient facilement démasquer de nombreuses irrégularités chez les représentants américains, y compris chez les membres du Congrès (Greenhaw, 1984, p. 96). De plus, les dirigeants corrompus, comme Noriega, seront recyclés en rebelles populaires combattant l'impérialisme américain. Par ailleurs, des intérêts canadiens ont aussi été impliqués dans ces enquêtes. Les banques canadiennes manipulent environ 80 pour cent des opérations bancaires des Bahamas et, au cours des dernières années, la Banque de Nouvelle-Écosse a frustré les applicateurs de la loi américaine en se conformant à la loi des Bahamas, c'est-à-dire en refusant de remettre les dossiers bancaires des clients soupçonnés de trafic de drogue. Incapable d'abolir la loi bahamienne, un tribunal américain imposa, aux succursales de la Banque de Nouvelle-Écosse aux É.-U., des amendes totalisant près de 2 millions de dollars pour les forcer à divulguer ces renseignements (Marsden, 1986). McGrath (1985) admet que les autorités américaines se font «plus insistantes» dans leurs demandes d'information aux institutions 
financières des lles Caïmans, des Antilles néerlandaises et des Bahamas, mais il rejette le blâme de cette agression extra territoriale sur les «trafiquants de cocaïne des Amériques».

Comme on ne s'attend pas à ce que les paysans péruviens s'apitoient sur le sort de riches Nord-Américains écervelés, on exporte la croisade anti-drogue jusqu'en Amérique latine sous prétexte que le trafic de drogues illicites ruine aussi les pays producteurs. Un représentant du Vénézuela a récemment averti les consommateurs de drogues canadiens qu'ils bouleversaient les «fragiles démocraties» de l'Amérique latine (Globe \& Mail, 15 jan. 1987). Il est vrai que ce type de trafic a des répercussions négatives dans les pays producteurs: violence, corruption et remplacement des méthodes traditionnelles de consommation (mâcher la coca) par de nouvelles méthodes plus dangereuses (fumer la cocaïne) (Economist, 28 nov. 1987 et 30 jan. 1988). Mais ces effets sont imputables à la politique gouvernementale et non à la demande des consommateurs : il suffit de comparer le café, qui est légal, à la cocaïne, qui est interdite. Mais regardons aussi le côté positif de la situation. La montée des paysans entrepreneurs signale un progrès démocratique puisque le système économique de la drogue ébranle le système actuel fondé sur les plantations, la concentration des propriétés terriennes et un prolétariat ouvrier. Healy (1986) rapporte que :

Dans un déroulement inhabituel, si on se fie aux normes historiques contemporaines de l'Amérique latine, pendant la rapide expansion commerciale qu'a connu la cocaïne au cours des huit dernières années, les petits propriétaires paysans ont conservé le contrôle de la matière première... Une autre caractéristique distincte... tient au fait que la technologie utilisée pour produire la feuille de coca ne dépend pas d'engrais pétro-chimiques importés.

Dans des conditions normales, l'investissement étranger arrive par des circuits officiels pro-occidentaux et son emploi crée un besoin d'aide supplémentaire, d'expertise américaine et de technologie. Mais dans les conditions artificielles créées par la croisade anti-drogue, l'aide que par inadvertance les prix du marché noir procurent, se rend en des lieux que gouvernements et sociétés commerciales ne peuvent contrôler. Il en résulte, d'une part, un modèle de développement relativement égalitaire qui ne va pas sans rappeler celui du Canada et des É.-U. dans les années 1800 , et d'autre part, un regain de popularité pour les producteurs de drogues qui subventionnent les travaux publics, la construction d'écoles, d'égoûts, de maisons et de sites de récréation destinés à attirer les employés dans un milieu compétitif (TIME, 7 mars 1988). 
Les autorités américaines affirment aussi que l'intervention en Amérique latine et partout ailleurs est justifiée pour prévenir la chute des gouvernements amis importants pour la sécurité des hémisphères. En fait, la menace que représentent les drogues illicites est née aux É.-U. et pourrait être supprimée du jour au lendemain en légalisant le commerce. Les sommes dépensées pour des substances prohibées pourraient, la plupart du temps, être éliminées ou perçues en impôts en légalisant possession et vente. Au lieu de cela, les gouvernements gaspillent des milliards à «chasser le dragon» de la destruction des cultures, et les É.-U. risquent de se retrouver, mis à part le Canada, entourés de gouvernements achetés ou intimidés par les trafiquants.

\section{OPTER CONTRE LA CROISADE ANTI-DROGUE}

\section{A. RÉFORME AU SEIN DES ÉTATS-UNIS}

Puisque les Américains achètent $60 \%$ de toutes les drogues illicites et financent plus de $90 \%$ de l'action policière contre le trafic, la légalisation des stupéfiants aux É.-U. marquerait une fin décisive à la croisade internationale. Mais peut-on envisager une telle réforme? Certains signes en laissent prévoir une. Pour la première fois depuis la levée de la prohibition sur l'alcool en 1932, des personnalités appartenant à diverses institutions (maires, membres du Congrès, policiers, avocats, journalistes et universitaires) entrent dans le débat et recommandent parfois une certaine forme de légalisation. ${ }^{8}$ Mais pourquoi la réforme n'est-elle qu'un projet lointain à l'heure actuelle et non pas dix ou vingt ans plus tôt, au moment où des commissions gouvernementales respectées préconisaient une réforme libérale à la fois au Canada et aux É.-U.?9 En raison des changements dans la configuration du conflit social. Les drogues illicites étaient une marque d'appartenance

8. D'éminents opposants à la guerre anti-drogue, parmi lesquels on retrouve Milton Friedman, économiste; Ethan Nadelmann de Princeton; Alan Dershowitz et Lester Grinspoon de Harvard; Thomas Szasz; David Boaz de l'Institut Cato et William F. Buckley, Jr. Amold Trebach de l'Université américaine de Washington ont fondé récemment la "Drug Policy Foundation» pour la promotion d'une réforme de la loi sur les drogues. Le maire de Baltimore, Kurt Schmoke, a demandé un débat national sur la légalisation des drogues et son initiative est appuyée par les maires de Washington et de Minneapolis et par au moins deux congressistes démocrates, Hoyer du Maryland et Stark de la Californie. Récemment, le débat sur la légalisation a fait la manchette du Washington Post, du Boston Globe et du New York Times. Le reportage principal de la revue TIME du 30 mai 1988 porte sur la légalisation des drogues. On lit dans cet article intitulé Thinking the Unthinkable que des représentants officiels sont «amèrement déçus» par la guerre anti-drogue.

9. Se reporter aux quatre études produites par la Commission d'enquête sur l'usage non-médical des drogues (1970-73) et publiées par Information Canada ainsi qu'à Report of the Select Committee on Narcotics Abuse and Control, 95e Congrès, première session, 1977. 
pour la jeunesse marginale et les activistes noirs (Lidz \& Walker, 1980). Avec le vieillissement de ces groupes, les drogues illicites sont plutôt devenues un problème de classe moyenne et de lieu de travail. Toutefois, de nouvelles drogues de ghetto comme le «crack» sont encore en cause. La démographie compte aussi pour beaucoup dans le remplacement de la génération Anslinger-Nixon-Reagan (les extrémistes pro guerre froide/guerre anti-drogue) par une génération du baby-boum qui dispose d'une certaine expérience personnelle des substances illicites. De plus, à mesure que cette génération vieillit, il y aura trop d'adultes et pas assez d'adolescents. Le nouveau modèle démographique aura deux conséquences significatives. Premièrement, l'attitude pro-sobriété et pro-santé propre à l'âge adulte répendra des idées plus restrictives sur les drogues, illicites ou autres. En même temps, la petite cohorte d'adolescents ne posera pas de problème social aux adultes (contrairement à la génération des années 60 ). Les adultes pourront ainsi se montrer plus ouverts à la rébellion et aux expérimentations des jeunes. À l'inverse, l'ancienne génération de politiciens a l'esprit étroit, quand il est question de diverses drogues, mais l'a plutôt libéral quand on parle de ses drogues favorites.

La peur de l'échec est une autre source de réforme. À l'origine, la politique anti-drogue était reliée aux réalisations américaines. Elle véhiculait donc une apparence de progrès. Mais aujourd'hui, après des décennies d'échec et d'hypocrisie officielle, la croisade anti-drogue peut être qualifiée de désuète et de mal planifiée. Les critiques fusent du côté des journalistes qui, durant les années 20 , se moquaient de la prohibition mais qui, jusqu'à récemment, avaient presque tous accepté la propagande officielle anti-drogue (National Review, 15 août 1986 et Globe \& Mail, 12 mars). Des sondages montrent que la majorité s'oppose à une réforme libérale des lois sur les drogues mais qu'elle pourrait rapidement tourner casaque si les dirigeants ajustaient leur tir. En effet la majorité connaît peu de choses sur les drogues illicites et en a donc une opinion très superficielle. D'ailleurs les renversements d'opinion soudains constituent plutôt la norme dans le domaine des affaires extérieures (Stupak, 1976). La diversité croissante des lois sur les drogues dans chaque État donne un autre coup d'éperon à la réforme fédérale. Certains États, comme l'Orégon et l'Alaska, ont déjà retardé le contrôle criminel de l'usage de la marijuana et une expérimentation plus poussée contribuera à mettre en évidence la futilité des mesures fédérales de contrôle sur les stupéfiants (Trebach, 1987).

Par contre, même si la légalisation des drogues semble logique, rien ne permet d'affirmer que le gouvernement fédéral américain agira 
logiquement. Établissant des parallèles historiques, Kennedy (1987) observe que beaucoup de nations au sommet de leur puissance militaire voient leurs fondements économiques décliner et leurs dirigeants tomber dans des aventures malencontreuses qu'ils ne peuvent se permettre. En expliquant ce phénomène, on explique pourquoi tous les États finissent un jour par décliner. Certains théoriciens offrent l'explication suivante: les groupes d'entreprises concentrés sont les plus nombreux à demander des législations. Ainsi, avec le temps et en l'absence de limites constitutionnelles efficaces, les lois spéciales servant des intérêts particuliers viennent à proliférer et nuisent à la société (Buchanan, 1975). Les citoyens perdent le contrôle sur les élus (Downs, 1957; Trebilcock, 1975) et les lois deviennent de plus en plus fascistes (Twight, 1975). Olson prédit que si elles disposaient de stabilité politique et de temps, toutes les sociétés se stratifieraient juridiquement en élevant des barrières de plus en plus hautes pour bloquer la concurrence. Les drogues en sont un exemple puisque les lois ont empêché les fournisseurs socialement marginaux de drogues tabous de concurrencer les producteurs dominants d'alcool, de tabac et de produits pharmaceutiques; de plus, le contrôle des drogues définies récemment comme «dangereuses» passait aux mains des professions puissantes et montantes que sont la police et la médecine. Au même moment, on interdisait la plupart des exportations de drogues venant de pays en voie de développement. On peut donc analyser tout le processus de répression anti-drogue en fonction d'une élite exerçant son influence sur les législateurs en vue de faire obstacle à la concurrence loyale. Ces théories et ces modèles historiques impliquent qu'une telle réforme est peu probable à moins d'une crise significative d'instabilité politique aux É.-U. Une autre possibilité serait qu'une réforme hors des É.-U. provoque une crise de concurrence à l'échelle internationale.

\section{B. RÉFORME HORS DES ÉTATS-UNIS}

L'ONU est une source peu probable de réforme, en raison du rôle prépondérant des représentants américains dans le processus international de contrôle des drogues. Ses études biaisées ne manquent jamais de se répandre en éloges sur les efforts des É.-U. et de blâmer le TiersMonde exportateur de drogue (McNicoll, 1983, p. 69). Pour des raisons similaires, les agences de contrôle de l'ONU n'osent pas réglementer les producteurs pharmaceutiques ni s'attaquer au détournement illégal de drogues «médicales».

Une réforme est plus probable dans un pays qui aurait avantage à se démarquer des É.-U. en matière de drogue, contrairement à ce que 
fait l'ONU. Jusqu'à maintenant, les autres pays industrialisés ont copié ou anticipé les détails juridiques de la croisade anti-drogue nord-américaine. Mais des signes de dissension commencent à poindre. Dans un récent éditorial du Criminal Law Journal d'Australie (1986) on pouvait lire :

Il semble que nous ayons maintenant besoin d'un équivalent du «Australian Act» qui nous rend indépendant de la Grande Bretagne pour rompre l'asservissement qui nous lie aux intérêts américains et qui nuit au développement d'une politique indépendante et rationnelle visant à règlementer la vente de drogue et à protéger la jeunesse... Lorsque l'on traite la consommation de stupéfiants de la même façon exagérée que la loi médiévale traitait la sorcellerie et l'hérésie, on encourage le vice que l'on veut supprimer.

André Fontaine, éditeur du journal Le Monde, exprime la même méfiance envers la politique étrangère américaine parce qu'elle semble fondée sur l'illusion que le code moral américain «est la valeur universelle et que les É.-U. ont le droit de le faire respecter» (Fontaine, 1987). Pourquoi la dissension au sujet de la drogue surgit-elle maintenant? Parmi les raisons plausibles, on retrouve le déclin relativement important de la domination militaire et économique américaine, le désir de parer à un revirement des Américains dans les guerres anti-drogue, les insatisfactions engendrées par les coûts de la croisade, et enfin, les avantages commerciaux que procure le fait de compter parmi les premiers pays à légaliser la drogue.

Après 1945, des pays comme la France et l'Australie ne pouvaient pas sérieusement résister aux politiques américaines qui accompagnaient l'aide américaine, les investissements et l'assistance militaire. Mais depuis les années 60, le relatif déclin de la puissance américaine laisse prévoir des divisions et des dissentiments au sein des alliés de la guerre anti-drogue. Au sortir de la Seconde Guerre mondiale, les ÉtatsUnis étaient la puissance mondiale suprême, avec un taux de production trois fois plus élevé que celui de l'URSS. Dans les années 50, ils produisaient $75 \%$ des véhicules motorisés de la planète et $50 \%$ de l'acier, tout en maittrisant la moitié des réserves financières mondiales. $\mathrm{Au}$ cours des années 60 , seulement $8 \%$ des producteurs américains avaient à se préoccuper de la concurrence étrangère alors qu'aujourd'hui au moins $70 \%$ doivent l'affronter (Reich, 1983). On remarque quelques signes de déclin: le pourcentage de pays en voie de développement qui alignent leurs monnaies à la monnaie U.S. est tombé de $60 \%$ à 25\% entre 1970 et 1987 (Economist, 19 sept. 1987). En 1955, la production américaine comptait pour $40 \%$ de la production mondiale; 
en 1980, elle n'en formait que $25 \%$ (Mack, 1986). Entre 1980 et 1986 , le déficit U.S. a augmenté de $600 \%$, plaçant les É.-U. devant le Brésil et leur conférant le titre de plus gros débiteur au monde (Thurow et Tyson, 1987). Voici quelques autres signes du déclin américain : ralentissement de la productivité, lourdeur de la bureaucratie, perte du leadership bancaire et technologique aux mains du Japon et agrandissement de l'écart entre les classes sociales (Morris, 1988). La guerre antidrogue est un exemple de malaise général causé par la mauvaise gestion économique d'un gouvernement (Calleo, 1987). En effet, les Américains se sont nui à trois points de vue dans cette guerre aux drogues: les consommateurs paient des prix exorbitants, les responsables gaspillent des ressources à combattre la production de drogue et rongent ensuite leur frein quand les trafiquants de drogues étrangers utilisent l'argent américain pour acheter des appareils, des véhicules ou de l'équipement fabriqué dans les pays asiatiques. L'obsession officielle des Américains pour «l'argent sale» ne peut constituer une riposte efficace au défi financier japonais. De même, la chasse aux sorcières contre les employés, les étudiants et les soldats consommateurs de drogues illicites ne constitue guère une réponse appropriée à l'incompétence des gestionnaires, aux universités moribondes ou à la lourdeur des institutions militaires.

Le relatif déclin de l'hégémonie économique américaine siginifie que les autres pays accepteront moins facilement les fardeaux de la croisade anti-drogue et que les É.-U auront plus de mal à acheter la coopération des dirigeants étrangers. Le déclin américain signifie aussi un alignement politique mondial plus fragmenté et une diversité qui inspirera des politiques plus indépendantes en matière d'alliances militaires, d'armes nucléaires, d'alliances anti-drogue, etc.

L'abandon de la croisade apportera des avantages commerciaux aux premiers pays capables d'innover. Pour commencer, la nation réformée deviendra une source unique de qualité : marque déposée de morphine, d'héroïne, de LSD, de cocaïne, de marijuana et ainsi de suite. Pendant les années 20, le whisky Canadian Club de Seagram a capturé et ensuite conservé une part appréciable du marché U.S. grâce à sa supériorité évidente sur les alcools élaborés clandestinement. De même, dans les pays légalisateurs, les entreprises deviendront chefs de file dans la transformation des drogues psychotropes et elles en retireront un avantage substantiel sur le marché U.S. grâce à leur marque déposée. L'industrie de transformation de la drogue se développera grâce aux débouchés connexes qu'offrent les produits chimiques, la transformation des aliments et la biotechnologie. On peut aussi 
s'attendre à voir une croissance du tourisme car beaucoup de gens seront alléchés par l'idée de goûter au «fruit défendu» dans un milieu sûr et réglementé.

On peut tenter de déterminer quel sera le premier pays à briser le carcan de la persécution quasi-religieuse dont font objet les consommateurs de drogue, mais ce ne sont évidemment que de pures conjectures. Parmi les candidats, on retrouve les pays déjà indépendants des É.-U., comme le Paraguay (Rosenberg, 1987) et les pays exportateurs, comme la Colombie et le Liban. Il y a aussi les petites démocraties indépendantes comme la Nouvelle-Zélande, l'Australie et la Suisse. Les entreprises canadiennes sont sans doute les mieux placées pour exploiter le marché américain mais le Canada est probablement trop vulnérable aux représailles américaines pour se lancer seul dans une course aux drogues.

Une réforme dans une démocratie industrielle aurait plus d'influence sur la politique américaine en raison des conditions d'expérimentation assez semblables. Les lois nord-américaines actuelles sur les drogues sont fondées sur deux axiomes erronés: 1) pour le bien de la société, la police doit tenir les citoyens loin des stupéfiants et 2) pour la santé de chacun, les médecins doivent empêcher l'auto-médication (Mitchell, 1986). La survivance de ces mythes sous-entend une limitation tolérante de la législation. Si une nation similairement constituée devait décriminaliser ou démédicaliser les drogues, il se produirait l'équivalent d'une réforme protestante et la fraude américaine sur la drogue serait dévoilée aux électeurs et aux groupes d'entreprises de la façon la plus dramatique et la plus probante qui soit. Rappelons un précédent: pendant les années 20 , de nombreux analystes américains prônaient l'adoption par le gouvernement de la «Solution du Québec» en vue de contrôler l'alcool par le biais de régies des alcools dans chaque État (Rowland, 1988). Dans un autre modèle, le contrôle de l'hérö̈ne en Angleterre est souvent comparé à celui du Canada et des É.-U. (Trebach, 1982).

Dans le cas d'une réforme qui aurait lieu hors d'une démocratie industrialisée, la réaction prévisible des É.-U. serait diffamatoire, alarmiste et antagoniste. Encore une fois, les liens canado-américains dans les années 20 constituent un exemple approprié. Au cours de cette période, le gouvernement fédéral américain exerçait des pressions sur le gouvemement canadien pour faire interdire l'exportation de «boissons alcooliques». MacKenzie King ignora cette requête jusqu'en 1929, délai qui en dit long sur les liens puissants qui unissaient alors le 
Canada et l'Angleterre. Avec le temps, King donna son accord au Canada Export Act (Loi canadienne sur l'exportation) de 1929, en partie à cause de promesses des Américains qui exempteraient le Canada de nouvelles lois protectionnistes. Il est intéressant de noter que malgré l'interdiction de King sur les exportations, le Canada ne fut pas exempté de nouveaux tarifs douaniers et que, trois ans plus tard, sous la nouvelle administration Roosevelt, les Américains mirent complètement fin à la prohibition (Rowland, 1988).

\section{CONCLUSION}

On dit que le commerce des drogues est, à l'heure actuelle, le plus grave problème relié au crime organisé. En fait, cette définition criminalisante est l'exemple classique d'entrepreneurs abusant des voies légales pour légaliser leur commerce de drogue et criminaliser celui de leurs concurrents. En fait d'abus, la véritable agression dans le domaine des drogues a été perpétrée par les puissances occidentales, principalement par les É.-U., aux dépens des cultures du Tiers-Monde. Quant à prédire le déroulement de la croisade anti-drogue, nous devrions envisager, d'une part, la possibilité d'une escalade dans les guerres civile et mondiale et d'autre part, la perspective de réformes pacifiques inspirées soit par des pays innovateurs hors des É.-U., soit par l'ajusternent des politiques américaines.

\section{BIBLIOGRAPHIE}

BARIDON, P. (1976), Addiction, Crime and Social Policy. Mass., Lexigton Books.

BATES, R.; LIEN, D. (1985), «On the Operations of the International Coffee Agreement», International Organization, 39: 553-559.

BLACKWELL, J. (1988), «Canada in a Global Setting: Notes on the International Drug Market», in J. Blakcwell \& P. Erickson, Illicit Drugs in Canada - A Risky Business. Canada, Nelson.

BONNIE, R.; WHITEBREAD, C. (1970), «The Forbidden Fruit and the Tree of Knowledge: An Inquiry into the legal history of American marijuana prohibition», Virginia Law Review, 56 : 972-1203.

BOYD, N. (1984), «the origins of Canadian narcotifs legislation : The process of criminalization in historical context", Dalhousie Law Journal, $8: 102-136$.

BRESLER, F. (1980), The Trail of the Triads - An Investigation into International Crime. London, Weidenfeld \& Nicolson. 
BUCHANAN, J. (1975), The Limits of Liberty: Between Anarchy and Leviathan. University of Chicago Press.

BURSTEIN, D. (1982), «The Deadly Politics of Opium», MACLEAN'S, Dec. 6, 1982 at 10-16.

CALLEO, D. (1987), Beyond American Hegemony: The Future of the Western Alliance. New York: Twentieth Century Fund.

CANADA (1973), Commission of Inquiry into the Non-Medical Use of Drugs. Final Report. Ottawa, Information Canada.

CHATTERJEE, S. (1981), Legal Aspects of International Drug Crontrol. The Hague, Martinus Nijhoff.

CLOYD, J. (1982), Drugs and Information Control. Westport, Conn., Greenwood Press.

Drug Enforcement: (1985), D. Westrate, «Drug Trafficking and Terrorism». (1983), F. Mullen, «Organized Crime and Drug Trafficking». (1982), W. Smith, «Drug Trafficking Today-Challenge and Response»; R. Caffrey, "The Strategy of Enforcement»; S. Cohen, «Health Hazards of Cocaine». (1978), C. Percy, «Angel Dust-Devil in Disguise»; E. Davis, «Modern Slavery».

DOWNS, A. (1957), An Economic Theory of Democracy. New York, Harper \& Row.

THE ECONOMIST : (1988), «Getting Gangsters Out of Drugs» Apr. 2, p. 11; «The Drug Economy», Apr. 2, p. 62; "Cocaine Killed him», Jan. 30, p. 32. (1987) "Looking Beyond America's Means», Sept. 19, p. 76; «Columbia - Stronger than the state», Nov. 28, p. 43. (1986), «A Hard-to-break Habit», May 24, p. 43; «Congress and White House on an Anti-drug High», sept. 20, p. 27; «Heroin Brings More Trouble», Dec. 20, p. 51.

EDITORIAL (1986), Criminal Law Journal, 10 : 117-118.

EITZEN, D.; TIMMER, D. (1985), Criminology : Crime and Criminal Justice. New York, Wiley.

EPSTEIN, E.J. (1977), Agency of Fear. New York, G.P. Putnam's.

Far Eastern Economic Review (1986), «Equal under the law», July 17 at pp. 26-27.

FAVRET, R. (1986), «Deadly Harvest», Globe \& Mail, Nov. 22 at D5.

FORT, J. (1969), The Pleasure Seekers: The Drug Crisis, Youth and Society. New York, Grove Press.

FONTAINE, A. (1987), «Beyond Wilson and Rambo», Foreign Policy, $65: 33$.

FREEMANTLE, B. (1985), The Fix : The Inside Story of the World Drug Trade. London, Michael Joseph.

GARRATY, J.; GAY, P. (1972), The Columbia History of the World. New York, Harper \& Row.

The Toronto Globe \& Mail, «Caution urged on prescription drugs at the border», Aug. 23, 1988, p. Al. «Report condemns U.S. drug war in Latin America», June 11, 1987, p. A13. «Moral Crusade - U.S. wins few skirmishes in was on drugs», March 3, 1986, p. Al. «Moscow joining with West in battling drug trafficking», Sept. 1, 1987, p. A17.

GOOBERMAN, L. (1974), Operation Intercept - The Multiple Consequences of Public Policy. New York, Pergamon Press. 
GOODE, E. (1984), Drugs in American Society. New York, Alfred A. Knopf, 2d. ed.

GREENHAW, W. (1984), Flying High - Inside Big-Time Drug Smuggling. New York, Dood, Mead.

GRINSPOON, L.; BAKALAR, J. (1976), Cocaine. New York, Basic Books.

HAQQANI, H. (1986), «Poppy bloom boom», Far Esastern Economic Review, July 10 , p. 42.

HEALY, K. (1986), «The Boom within the Crisis: Some Recent Effects of Foreign Cocaine Markets on Bolivian Rural Society and Economy», in D. Pacini \& C. Franquemont, eds. Coca and Cocaine. New York, Cultural Survival.

HELMER, J. (1975), Drugs and Minority Oppression. New York, Seabury Press.

HESS, A. (1965), Chasing the Dragon - A Report on Drug Addiction in Hong Kong. Amsterdam, North-Holland Pub.

INCIARDI, J. (1986), The War on Drugs - Heroin, Cocaine, Crime and Public Policy. Palo Alto, Mayfield Pub.

JOHNSON, W. (1986), «Moral Crusade - U.S. wins few skirmishes in was on drugs», Globe \& Mail, Mar. 12, p. A1.

KENNEDY, P. (1987), The Rise and Fall of the Great Powers : Economic Change and Military Conflict, 1500-2000. New York, Random House.

KAPLAN, J. (1970), Marijuana : The New Prohibition. New York, World.

KLINE, D. (1987), «How to Lose the Coke War", The Atlantic, May, p. 22-27.

LAMOUR, C.; LAMBERTI, M.S. (1972), The Second Opium War.

LEE, H. (1963), How Dry We Were - Prohibition Revisited. New Jersey, Prentice Hall.

LEE, R. (1985), «The Latin American Drug Connection», Foreign Policy, 61 :142.

LIDZ, C.; WALKER, A. (1980), Heroin, Deviance and Moraliry. London, Sage Pub.

MACLEAN, S. (1987), «Crackdown on the Montreal Connection», Apr. 6, p. 45. (1982) «From a smuggler's paradise comes hell», Nov. 8, pp. 12-20. (1979) «The War on Drugs", Apr. 2, pp. 25-30.

MACK, A. (1986), «The Political Economy of Global Decline: America in the 1980s», Australian Outlook, 40-11.

McCOY, A. (1972), The Politics of Heroin in Southeast Asia. New York, Harper \& Row.

McNICOLL, A. (1983), Drug Trafficking : A North-South Perspective. Ottawa, NorthSouth Institute.

MACFARLANE, B. (1986), Drug Offences in Canada. Toronto, Canada Law Book.

McGRATH, P. (1985), «Drugs and Tax Havens», Glohe \& Mail, Aug. 24, p. A7.

MARSDEN, W. (1986), «Laundering "narco-dollars» in the Bahamas», Toronto Star, Jan. 26, p. G1.

MILLS, J. (1986), The Underground Empire: Where Crime and Governments Embrace. New York, Doubleday. 
MITCHELL, C. (1986a), «A Justice-Based Argument for the Uniform Regulation of Psychoactive Drugs», McGill Law Journal, $31: 212-263$. (1986b), «Deregulating Mandatory Medical Prescription», American Journal of Law and Medicine, 12: 207-239.

MORALES, E. (1986), «Coca and Cocaine Economy and Social Change in the Andes of Peru», Economic Development and Cultural Change, 35 : 143-161.

MORGAN, H.W. (1981), Drugs in America: A Social History, 1800-1920, Syracuse University Press.

MORRIS, D. (1988), «United States starts looking like a Third World country», Globe \& Mail, Mar. 10. p. A7.

MULLER, E. (1985), «Dependent Economic Development, Aid Dependence on the United States, and Democratic Breakdown in the Third World», International Studies Quarterly, $29: 445$.

MUSTO, D. (1973), The American Disease: Origins of Narcotic Control. New Haven, Yale University Press.

MURRAY, G. (1988), «The Road to Regulation: Patent Medicines in Canada in Historical Perspective» in Mllicit Drugs in Canada Supra.

MURRAY, G.; SMART, R. (1985), «Narcotic Drug Abuse in 152 Countries : Social and Economic Conditions as Preditors», International Journal of Addiction, 20: 737.

MURRAY, G.; SMART, R. (1980), The History and Rationale for the Convention on Psychotropic Substances" in Psychotropic Substances and their International Control. Toronto, Addiction Research Foundation.

NATIONAL REVIEW (1987), «Exporting the Drug War», May 12, p. 38. (1986), «Bolivia and the Home Front», Aug. 15, p. 17.

OLOSON, M. (1982), The Rise and Decline of Nations. New Haven, Yale University Press.

PAN, L.; BRUUN, K. (1979), «Recent Developments in International Drug Control», British Journal of Addiction, $74: 141$.

PASTOR, R. (1986), «Does the United States Push Revolutions to Cuba? The Case of Grenada», Journal of Interamerican Studies and World Affairs. 28 : 1-34.

RAY, O. (1978), Drugs. Sociery and Human Behaviour. St-Louis, C.V. Mosby. REICH, R. (1983), The Next American Frontier. New York, Basic Books.

ROBERTSON, F. (1977), Triangle of Death - The Inside Story of the Triads. Toronto, General Pub.

ROSENBERG, T. (1987), «Smuggler's Paradise», The New Republic, June 8.

ROWLAND, R. (1988), «Free trade's nail in the temperance coffin», Globe \& Mail, Sept. 2, p. A7.

RUBLOWSKY, J. (1974), The Stoned Age - A History of Drugs in America. New York, Putnam's.

SATINDER, K. (1980), Drug Use : Criminal, Sick or Cultural. New York, Libra Pub.

SCIENCE (1975), «Drug Abuse 1975: The «War» is Past, the Problem is as Big as Ever», Nov. 14, p. 638.

SILVERMAN, M.; LEE, P.; LYDECKER, M. (1982), Prescriptions for Death. Berkely, University of California Press. 
SOLOMON, R.; GREEN, M. (1982), «The First Century: The History of Non-Medical Opiate Use and Control Policies in Canada, 1870-1970», Univesity of Western Ontario Law Review, 20 : 307-336.

STEPHENS, R. (1987), Mind-Altering Drugs - Use, Abuse and Treatment. New York, Sage Pub.

STRUG, D. (1986), «The Foreign Politics of Cocaine : Comment on a Plan to Eradicate the Coca Leaf in Peru", in Coca and Cocaine, Supra.

STUPAK, R. (1976), American Foreign Policy - Assumptions, Processes and Projections. New York, Harper \& Row.

SZASZ, T. (1974), Ceremonial Chemistry : The Ritual Persecution of Drug Addicts and Pushers. New York, Anchor Press/Doubleday.

TAYLOR, A. (1969), American Diplomacy and the Narcotics Traffic, 1900-1939. Duke University Press.

THUROW, L.; TYSON, L. (1987), «The Economic Black Hole», Foreign Policy, 67 : 3-7.

TIME (1988), «Wanted Noriega», Feb. 15, pp. 14-15. «Still in charge», and «Drug Thugs», Mar. 7, pp. 34-41. "Tears of Rage», Mar. 14, pp. 16-18. «The Big Squeeze», Mar 21, pp. 34-36. (1986), «Striking at the Source», July 28, pp. 10-12. (1983), «Battle of the Warlords», Jan. 17, p. 28. (1982), «The Great Opium War», March 1, p. 28. (1979), «The Columbian Connection», Jan. 29, pp. 16-24.

TREBACH, A. (1987) The Great Drug War. New York, MacMillan. (1982) The Heroin Solution. New Haven, Yale University Press.

TREBILCOCK, M. (1975), «Winners and Losers in the Modern Regulatory System Must the Consumer Always Lose?. Osgoode Hall Law Journal, $13: 619$.

TWIGHT, C. (1975), America's Emerging Fascist Economy. Mass. Arlington House.

U.N. MONTHLY CHRONICLE (1984), Vol. 21, no 10/11, pp. 62-64.

U.S. NEWS AND WORLD REPORT : (1987) «Columbia to drug lords : Enough is enough», Jan. 19, p. 12. «Narcotics: Terror's New Ally», May 4, pp. 30-37. (1986) "American Drugs», July 28, pp. 48-55. "Stop drugs at source», Aug. 25, p. 19." Fighting the drug war from the trenches», Sept. 8, p. 20. «The terror of illicit drugs», Sept. 8, p. 69.

WADDELL, I. (1970), «International Narcotics Control», The American Journal of International Law, 64 : 310-323.

WALKER, W. (1981), Drug Control in the Americas. University of New Mexico Press.

WISOTSKY, S. (1986), Breaking the Impasse in the War on Drugs. Westpon, Conn., Greenwood Press.

WHITE, L. (1988), Merchants of Death: The American Tobacco Industry. Toronto, MacMillan.

ZINBERG, N.; ROBERTSON, J. (1972), Drugs and the Public. New York, Simon \& Schuster. 